\title{
Undergraduate Students' Stress, Anxiety and Depression Remote Health Monitoring System
}

\author{
Mitchelle Liaw Ai Wei, Akeem Olowolayemo \\ Dept. of Cognitive Sciences and Human Development, Universiti Malaysia Sarawak, Sarawak, Malaysia. \\ Dept. of Computer Science, KICT, International Islamic University Malaysia. \\ akeem@iium.edu.my
}

\begin{abstract}
Mental health such as stress, anxiety and depression have been on the rise in recent time among different groups of people in the emerging challenging society and especially among university students. University is a place where most adolescents would experience a change phase in life, discovering their identity as well as experiencing several challenges. All these pressures might cause students to suffer from anxiety or stress or in some cases severe depression, which could lead them to isolate themselves from other people, or to have poor academic performance or very low self-esteem as well as dropping out from the universities. This is quite a dangerous trend because if such students are not treated early, the chances for their situation to worsen or ultimately attempting suicide would be high. Several technologies have been utilised to help assist clinicians and patients to confront and help mitigate health problems. Technologies such as eHealth, or mHealth which utilises smartphones and remote patient monitoring systems (RPMS) have been widely used in the healthcare and are proven to be efficient and quite reliable to help those who suffer from health issues. In this project, a remote mental health monitoring system (RMHMS) has been proposed to enable lecturers as well as university administration and therapists/counsellors to track students' level of stress, anxiety and depression and swiftly engage those who suffer from stress, anxiety and depressive symptoms. Students who suffer from severe stress, anxiety symptoms and depression, will be given more attention by the university's administrations based on the information available from the remote mental health monitoring system. The proposed system will help facilitate early interventions and treatment such as referrer to counselling sessions or seeking psychiatric help. The system equally incorporates alert systems to students' friends or families and their caregivers to intimate them of their situation too so that they may help the students to overcome their mental health issues before getting worsened.
\end{abstract}

Keywords-Mental Health, eHealth, mHealth, Remote Patient Monitoring Systems (RPMS), Remote Mental Health Monitoring Systems (RMHMS), University students.

\section{INTRODUCTION}

In recent times, more and more people are suffering from anxiety, severe stress, and depression. Mental health has become a pervasive issue especially in the universities around the world. Depression is the major cause of severe mental health problems and may cause students' performance in academic, socialization and self-care to deteriorate. One of the first symptoms of those suffering from depression is that they may begin to stop going or attending their classes as well as meeting their classwork and assignments' deadlines. Several university students with severe depression have been shown to attempt suicide in recent time, according to Anxiety and Depression Association of America [1].
One of the precursors to depression is anxiety. Anxiety is human body's natural reaction to stress [1]. People who suffer from anxiety have the feelings of tensions, thoughts that are filled with worries and they may sometimes experience physical changes for example, increase of blood pressure. They usually have some unwanted thoughts too that are disturbing which keep reappearing in their mind and they might try to evade certain states because of worries. Evading situations that provoke anxiety only creates immediate relief from the anxiety which is a short-term solution. One can develop an anxiety disorder if these feelings of anxiety last for more than six months and hinder their lives [1]. 
Recently, the level of anxiety among university students have been on the rise due academic pressure, relationship's problems, financial status or influence from the environment around them [2]. Recently, the shocking news of a 20-year-old university student who committed suicide by jumping off a building in Seremban, Negeri Sembilan, due to depression because of intense pressure to excel in her studies demonstrates this growing problem[3]. Thus, majority have started to question the sudden rise of students suffering from mental health, attempting suicide, and harming themselves. Many factors that cause students to succumb to mental health problems include not being able to adapt themselves to the sudden change of environment in university, or cope with the incoming challenges, hostile classmates, loneliness as well as academic pressure. A study done to assess 506 students from four universities in Klang Valley with the age range from eighteen to twenty-four years old showed that the prevalence of the students suffering from moderate depression, anxiety and stress are very high [3]. It was said that 1 in 5 university students are suffering with anxiety [4]. A related study shows that more than half of the undergraduates in Hong Kong suffer from anxiety which is probably caused by immense academic pressure and cost of study [5], [6]. Similarly in [7] it was found that about one in six of the students had been confirmed suffering from anxiety in the US. The warning signals and symptoms of anxiety include isolation from daily activities, overeating or poor eating habits, alcohol or drugs abuse, online addictions, changing behaviours or emotions, and thoughts of committing suicide. Anxiety can be treated but majority of the students do not have the courage to admit that they suffer from anxiety and to seek help from professionals, friends or family members [2]. Malaysian health ministry revealed a decline state of mental health especially among Malaysian university and college students. Experts have cited anxiety and depression as the main causes of mental health problems among students although not ruling out the influence of drugs as a factor. Additionally, untreated state of mental health problems such as anxiety could lead students to isolate themselves, developed schizophrenia and have the thoughts of committing suicide [8].

Technology usage in healthcare has been on the rise and widely used nowadays. Remote patient monitoring (RPM) is a technology developed for healthcare different from using the traditional method but instead uses advancement in information technology such as mobile technology, sensors and internet of things to monitor and provide care as well as obtain patients' data for further investigation and monitoring. Remote Patient Monitoring (RPM) enables clinicians to communicate with patients as well as patients' family or friends who are essential to bringing success to the patients' treatments [9]. RPM is linked to the patients' compatible monitoring devices such as smartphones wirelessly to document patients' health data such as tracking their activities or monitoring their blood pressure. RPM could then be used to analyse the data obtained and provide real-time updates directed to the clinicians, families, or friends to be aware of their health conditions. RPM technology can be utilised to be able to detect symptoms or signs earlier, thus reducing the risk of aggravating the patients' conditions. This strategy has the potential to help in improving patients' health as it provides active interventions for them [10], [11]. Health informatics, eHealth as well as mHealth, concerns gaining, keeping, retrieving, and utilizing of healthcare information to promote better association between patients and clinicians in a digital format[12][13][14].

Studies of utilising technology for students' depression, stress and anxiety management system using mobile application are still quite few. The main aim of this study is to research how a remote health monitoring system can be utilised for better management of undergraduate students' stress, anxiety, and depression. The proposed remote mental health monitoring system is intended to enable the universities' administration in charge of students' affairs, counselling units and students' mental health therapists to track students who may have high level of stress, anxiety and depression by checking on their classes' attendance, participation in class, assignments' submission or responses to messages send to those students. This allows help to be given to those students who suffer from anxiety, severe stress, or depression to avoid undesirable circumstances. Moreover, this study also incorporates the Depression, Anxiety and Stress Scales (DASS 21) scales [15] so that students can be accessed using the scales early on in their study as well as at critical times within the semesters to determine their level of anxiety, stress and depression using the health monitor application. Students would not have to search for the scales to check their mental health states on different websites but utilise the application while observed critical results are transmitted to the in-charge administrative officers, counselling units and specified students' guardians. These students will be monitored attentively while at the same time comparing with their academic performance such as grades, submission of 
assignments and attendance in class. If these criteria plunge below expectations, the administrators are expected to take immediate actions such as scheduling a meeting with the student to discuss the problems that they are facing while necessary interventions are proposed. This is to make sure that they are aware and check-up on the students' conditions are carried out swiftly to avoid any unwanted situations. The expected outcomes of this remote health monitoring system are that students will be able to determine their level of anxiety, stress and depression by answering the DASS-21 questionnaires, better observations of students' mental health signs and symptoms as well as provisioning of faster interventions to the students. Consequently, the envisioned goal of this study is specifically to ensure that students who might have severe anxiety, stress and depression will be detected earlier and overall better management for such students can be provided.

\section{LITERATURE REVIEW}

This section focuses on the issues related to students' stress, anxiety, and depression remote health monitoring system as well as relevant past studies.

\section{A. Students' Stress, Anxiety and Depression Remote Health Monitoring System}

Majority, around $90 \%$, of physicians use smartphones or tablets in their everyday professional tasks [16] including to share the patients' data, these mobile applications are linked with the electronic health records (EHRs) which enhance management and add value to caregiving. These applications allow the relationship between patients and physicians to be enhanced, associating patients with peers, provide online communities for patients to take part in their own care and to supply helpful data regarding patients to both the patients as well as the physicians. A study in 2015 by World Health Organization (WHO) involving fifteen thousand mHealth applications in which $29 \%$ of them were focusing on mental health diagnosis, treatment or support demonstrate an increase in attempt to utilise mHealth in mental health care. Aside from WHO, UK's National Health Service (NHS) and U.S, National Institute of Mental Health (NIMH) have also revealed that mental health applications are profitable and measurable solutions for mental health treatment gap [17]. Clinical care provision such as instantaneous crisis intervention, prevention, diagnosis, preand post-treatment and therapy are stages expected in mental health management system. The key plan is to lessen the widespread presence, time span and burden of depression, early discovery, and treatment of depressive symptoms. since depression mostly occurs during adolescence to early adulthood. However, majority do not or are reluctant to seek help from professionals early on when depressive symptoms become noticeable while offering excuses such as expensive cost, limited acquisition of help and the stigma among people regarding mental illness. Thus, to assist them in overcoming these barriers by providing an approach to immediately access treatment and decrease their depressive symptoms, eHealth technology can be handy by replicating face-to-face treatment, admissible to end users, benefits in term of cost, anonymity, supply of helpful features which may increase treatment experience and end results as well as functionalities for changing the intervention experience are eHealth's actions to improve intervention and management of medical disorder [18].

\section{B. Anxiety among Freshman Students}

Students might experience a new kind of anxiety upon getting into the university for the very first time. This new anxiety may result from adaptation to a new environment, separation from family, academic pressure, financial burden, and changing responsibility. These challenges can thus evoke anxiety symptoms. Anxiety symptoms can be noticed when one feels anxious and worn out for no known reason. One may feel restless, while mind maybe preoccupied with several strange thoughts. Those undergoing transition phase from teenagers to adolescents may equally suffer from anxiety due to unfamiliar environment which they need to adjust, accept, and be accepted. Not only that, high expectations set on the students by their parents may also affect them, thereby contributing to experiencing anxiety. Examination or academic pressure is one of the factors that contribute to students developing anxiety [19].

Family background has been noted as a major cause of stress among adolescents coupled with experience in middle school. The growth of a child is determined by the parents' roles in their early developmental stages. Unfortunately, these stages are the times when the gaps between parents and their teenage children widens. Consequently, teenagers are often seen to develop disobedient attitudes to gain parents attention because they do not receive much love and attention from their parents unlike when they were younger, especially when they face challenges at school. In the study conducted by [20], it was shown that even though working mothers who have higher education are more sensitive and attentive 
towards their children, however, their children might be lonely and have low intelligence. Besides, poor and low family background contributes to high stress scores among adolescent students than those with good family background. However, working mothers whether from welloff family or poor family background contribute to higher stress in adolescent students than those non-working mothers from well-off or poor family background. Thus, it was concluded that adolescent students' stress is affected by their family background [20]. Adolescents are equally impacted by their environment, and hence stress level can be lower if a pleasant and heathy environment is provided in schools as well as within the family.

As stated by [21] adolescents might regard college life as the most unforgettable and memorable memories where they are able to enjoy the lively atmosphere, meet new friends as well as to get involve in academic and co-curricular activities that can help shape the adolescents into adulthood. From this study, factors which contribute most to stress among college students include academic stress resulting from high family expectations, teachers' expectations, competition with other students and huge amount of coursework loads. Family expectations is considered highest at $52.7 \%$ followed by teacher's expectations at $32.4 \%$ contributed the most to causes of academic stress among college students. Another important contributing factor is financial stress that includes no financial support from their families, their money is being controlled by parents, poor budget management as well as limited money for personal expenses. The study found an association between academic stress and social as well as financial stress. Students' academic performance would be low if they have difficulty in socializing and vice versa. It is shown that their academic performance would also be affected if they have financial stress.

Authors in [22] states that psychological, academic and social shock could probably affect the adaptation of students from school atmosphere to university atmosphere. This is due to the difference of the educational system and settings. Adaption causes the students to encounter numerous new changes such as different ways of teaching, different academic demand as well as meeting new people. Students might suffer from different types of stress which can have negative effect on their mental health and academic performance because of all the new, different changes they experience in university.

The study in [23] expressed that self-discovery, selfreliance, social expectation and peer pressure mostly happen during teenage years. Mental disorder can be found in one of four teenagers whereas sixteen to twenty-four years old with the percentage of $9 \%$ suffer from high to very high level of psychological disorders. Depression is often higher in teenagers who do not receive much love and attention from their family, agonized domestic violence or abuse and poor relationship with their family members. This is equally supported by earlier study by [24], based on the DASS-21 questionnaire to assess 242 adolescent students belonging to affluent families as well as linkage with diverse sociodemographic of the students. This can be supported by another research carried out by [25], where he mentions that women suffer from major depression is higher than men. Furthermore, with respect to academic factors, the DASS scores were high for depression, anxiety, and stress in adolescent students in tenth and twelfth classes whereas those in ninth and eleventh classes reported to have low score for depression, anxiety and stress. Thus, this research proves that depression is common in teenagers and suggested that effective methods should be provided in schools by the counsellors to help the teenagers in reducing depressive symptoms. The prevalence of depression among university students was found to be around 1 in 3 students or roughly, $33 \%[26]$. This is because freshmen normally stay far away from their families [27].

Stress and depression have powerful association whereby majority of people suffering from depression encountered stressful situations beforehand. Those who have high level of stress are prone to develop depression, decreased level of confidence in learning and poor study practice as well as socializing. These factors affect students' academic performance [28]. In a previous study to observe whether students have mild symptoms of depression, lecturers were able to notice students' attitudes and monitor their grades [29].

In another related study, [30] conducted a research on 1,023 university students in Malaysia within the age range of eighteen to twenty-four years old using the Centre for Epidemiological Studies Short Depression Scale (CESD-10). In this research, it is shows that $4.4 \%, 25.0 \%$ and $29.4 \%$ of the university students are experiencing severe, moderate, and minor depression respectively. Female students are reported to experience depression with an overall percentage of $53.5 \%$ while that of the male students is around $46.5 \%$.

One of the well-known healthcare practices is eHealth. A study carried out by [31] discussed the use of eHealth to promote physical activities in patients with mental health 
conditions. Physical activities can improve mood and sleep, reduce stress as well as to strengthen self-esteem. Patients with mental health issues normally have inadequate level of physical activity.

According to [32], mental disorder may have a negative consequence in the lives as well as on the friends and family of the patients. Those who have been diagnosed of mental disorder and committed suicide is around $90 \%$ in the United States. Nevertheless, those who receive treatment for such mental illness are less than $40 \%$. The reasons they do not seek for professional help including attempting to find their own solution to the problem, encounter situational obstacles as well as financial or costs obstacles. A mobile phone is always carried by anyone and hence, provide a means to collect data automatically serving as a wearable sensor platform located in the users' pocket. Even, it has been shown that the quality of data gathered using questionnaire incorporated in mobile phone is higher than using paper-based questionnaires.

\subsection{Remote Patient Monitoring and mHealth}

The study conducted in [33] described that mobile health (mHealth) and eHealth might have the chances to reach and help patients with major mental health. Specifically, smartphones, Short Message Service (SMS) or PDAs are the mobile interventions commonly utilised. 11 out of their 46 studies, focused on illness self-management and relapse prevention implemented using an mHealth application, Health Buddy. Health Buddy is an automated home messaging device targeted at three separate evaluations of the Information technology Aided Relapse Prevention Programme in Schizophrenia as well as a self-managing bipolar disorder using an online platform. Utilising this system was able to reduce the number of hospital admissions as well as emergency room visits by patients. Not only that, the severity of mental health could also be decreased using mobile interventions. Maintaining medication intake or treatment schedule is also done using mobile intervention whereby SMS intervention was used to deliver automated text message that serve as reminders for patients to consume their medications or to attend consultation with doctors or clinicians. This SMS interventions provide benefits for the patients to be reminded to attend the scheduled consultations on time for better management of mental health issues [33] and mHealth benefits and usage in medical care to help the patients was also studied in [34] and greatly help This information gained will then be sent and shared to the physicians or clinicians to allow easy tracking. Some mHealth is also designed to supply one-touch contact to an appropriate support hotline for the patients if there is an identification of important negative items or suffering by the patients' self-assessment. Audio recording of consultation sessions is also available for patients to review and smartphones are the perfect device to record high quality audio and video. Clinicians can also gain benefits from the audio or video recording of the sessions which the videos can be reviewed numerous times to make sure good treatments are provided. Other than audio or video recording, smartphones nowadays offer a real-time twoway communication capability which is video camera. This video camera allows patients and clinicians to have communication using video camera in real-time to obtain behavioural health information that is flexible and not expensive. Accessing databases and other clinical data is also provided by smartphones. Smartphones enable external hardwire device such as biofeedback to be connected. Biofeedback is very useful in monitoring physiological signals and it has been integrated into applications whereby it can be used to manage health problems such as stress, headache and hypertension. Therefore, the use of smartphone in mHealth have been recommended as it has been proven that it contributes advantages and benefits to both the clinicians and patients as well [34].

The study in [35] listed several common mHealth and ICT applications in the healthcare area. An example of the application is client education and behaviour change communication, to change the behaviour, attitude and to enhance the knowledge of the patients using sensor technology that can examine patients' health with the connection of 2-lead electrocardiograms (EGGs) namely AliveCor to the mobile phones. It allows clinicians to look and evaluate cardiac health.

Using the integration of pharmacotherapy such as medicine and cognitive-behavioural therapy (CBT), lots of mental disorders problems can be treated. This leads to aiding the patients on the ways to change their behaviour through medical observation without the interruption from the clinic [32]. Moreover, Gravenhorst et al., [32] also mentions that reminders, conversation and distant consultation can be done easily with smartphones and gave a general architecture of eHealth applications, namely; a server of data storage and a web-portal for browser-based access Furthermore, Kuhn et al. [36] conducted a study regarding self-management tools for patients who suffer from post-traumatic stress disorder to try out and evaluate self-management tool, found that it can improve caregiving. Ehrenreich, et. al [37] equally evaluated the effect of 
smartphone in psychiatric treatment among smokers who wanted to quit smoking and patients with anxiety.

Ambient intelligent system has also been proposed to aid patients with depression using a smartphone application as well as web-based behavioural activation intervention according to Harrison (2011). An association between their mood and the activities they take part can be shaped using the daily mobile monitoring to further motivate the patients. The system will then amylase their behaviour. Reminders, advises, feedback and suggestion are then sent to them for the patients to increase their mood.

"myCompass" is another system for patients suffering from mild-to-moderate stress, anxiety and depression developed by the Black Dog Institute in Australia. myCompass is a self-monitoring and self-management which is provided on users' smartphones and computers using the Internet. It offers plenty of interactive and adjustable functions. Moods, symptoms and behaviour can be tracked by the patients. Not only that, but patients are also able to gain feedback about their monitoring in a graphical method, study more about mental health and obtain helpful advices in helping themselves to get better. CBT-based self-management modules are also provided in the application whereby patients can select the type of modules they want to use or otherwise, the system will automatically select the applicable modules for the patients based on the questionnaires that they have taken [18]. Malasinghe, et al. [39], remote monitoring, real-time and quick detection of illness has become very well-known as they have moved to the world so quickly. Remote monitoring which means monitoring patients from a very far distant or outside of hospitals using technology has its own advantages. Thus, the advantages of using remote monitoring is that it enables detection of illness to be done as early as possible, potentiality to monitor patients consistently, stop the illness or conditions before it gets worse, cost-effective, decrease the number of hospital visits, provide precise data or readings of patients' health and provide emergency medical care. Data acquisition system, data processing system, end-terminal at hospital and communications network are the essential aspects of a remote monitoring system.

This section highlights the issues, past studies as well as current studies related to this study. Based on this section, the prevalence of university students suffering from stress, anxiety and depression can be seen. Furthermore, a few of the related technologies such as ehealth, mhealth and remote patient monitoring have been examined.

\section{METHODOLOGY}

This section explores the current manual process used in the faculty and the methodology proposed to create the remote health monitoring and management system.

\section{A. Current Manual Process in the Faculty}

The current manual process in the faculties of most universities concerned about mental health can be broken down into several steps. First of all, there may be lecturers' responsibilities to pay attention to information about students in their classes, specifically, students' attendance, grades, submission of assignments and their progress in class. If the students' progresses, attendance and submission of assignments are abysmally low, the lecturers are obliged to inform the Admin-in-charge or Student Welfare Officer or Deputy/ Dean of Students' Affair via email, phone call or message. Student Welfare Officer (SWO) then should reach out to the students to have discussion about the challenges that the students are facing that result in their low performance. If the students do not respond to the Admin-in-charge's calls or messages, their peers or families/guardians are contacted to ascertain their statuses or whereabout. Finally, Admin-in-charge would strive to help the students as much as possible based on their findings. However, if their conditions are quite extreme, they would be referred to the counsellors or psychiatrics for further check-up.

\section{B. Modelling the Student's View}

The first user requirement for this system is for users (university students) to be able to determine their levels of emotional states (stress, anxiety and depression) by answering the DASS-21 scale implemented in the remote health monitoring system. The DASS-21 assessment score is presented in table 1 . The first part of the mental health management system requires the students to respond to Depression, Anxiety and Stress Scales 21 (DASS 21) at the start of their programme and at critical points during the semesters and all the way through the course of their study until graduation. The instrument used for students to determine their level of stress, anxiety and depression is DASS-21 assessment. Users will be able to see their results displayed on the interface. Users will also be able to receive notifications of feedbacks or messages if necessary, to keep track of their health. As for the university SWO or faculty's administration or counsellors, they will be able to monitor those students who have high level of stress, anxiety or depression. 
TABLE I

DASS 21 ASSESSMENT SCORE

\begin{tabular}{|c|c|c|c|}
\hline & Depression & Anxiety & \multicolumn{1}{c|}{ Stress } \\
\hline Normal & $0-9$ & $0-7$ & $0-14$ \\
\hline Mild & $10-13$ & $8-9$ & $15-18$ \\
\hline Moderate & $14-20$ & $10-14$ & $19-25$ \\
\hline Severe & $21-27$ & $15-19$ & $26-33$ \\
\hline $\begin{array}{c}\text { Extremely } \\
\text { Severe }\end{array}$ & $28+$ & $20+$ & $34+$ \\
\hline
\end{tabular}

They will be able to send messages or appropriate interventions or feedback to the suspected students and if the students do not respond to them, an automated message will be sent to their families, friends or peers to alert them about the students' conditions.

DASS-21 is a three self-report scales to measure the emotional states of depression, anxiety and stress. These scales consist of 7 items. Each scale is used to assess certain criteria. The total score would determine the students' emotional states whether they are suffering from any of depression, anxiety or stress. It is hoped that incorporating this will enable easy identification of the students who might likely be suffering from any of the aforementioned mental illness. The features of the student's main page after registration into the system can be seen in figure 1 . The DASS-21 is the first item pushed to the students to respond after registration and subsequently at different critical intervals during their studies. The proposed system was prototyped using Thunkable. Thunkable is an online platform to build mobile apps, either for Android or iOS.

The student's flow on the remote mental health monitoring and management system is presented in figure 1. The first task is to respond to DASS-21 Assessment as well as respond to SWO's notifications when they are seen. After meeting with the SWO, they might be referred to the counsellor, therapists, or psychiatrist. When referred to one of counsellor, therapist, or psychiatrist, they continue to receive notification on their sessions, while follow up reports are sent to the SWO as well.

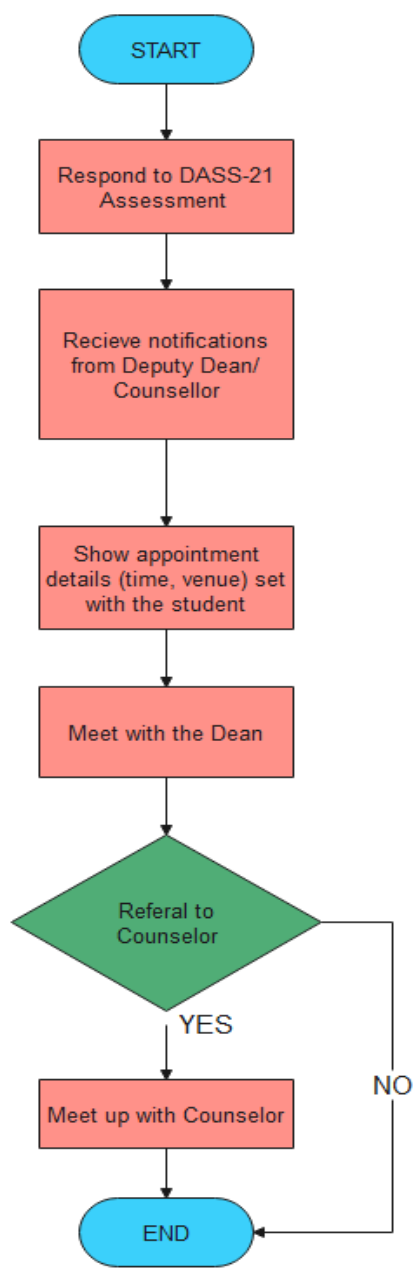

Fig. 1 Student's Flowchart

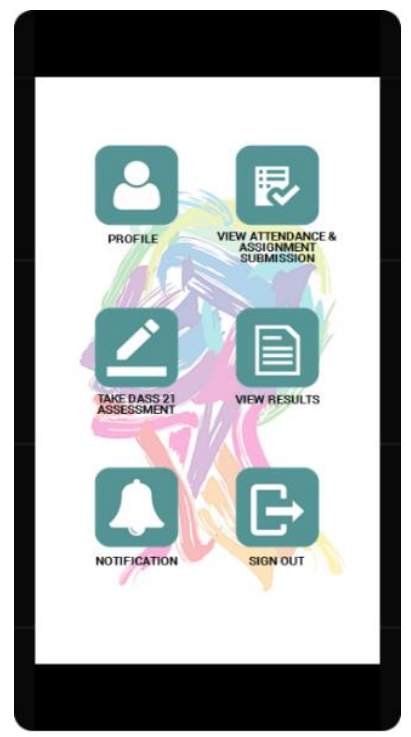

Fig. 2 Student's Main Interface after Registration 
In case, the student specified a trusted family member or guardian or next of kin, reports adequate for their needs can also be sent to them.
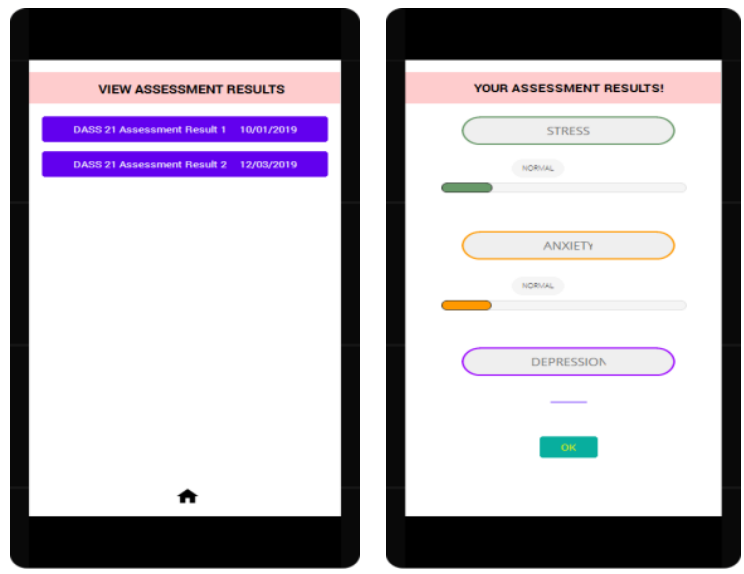

Fig. 3 Student's DASS-21 Results

However, if there is no mental health issues, then appropriate assistance such as financial aids may be recommended. On the remote system, the students main page contains the following dashboards, viz; Dass-21 Assessment and DASS-21 Scores (View Results), and a summary of percentages of student's metrics such as attendance, submission of assignments and participation in class. This can be seen in figure 2. These are important indicators of students focus and probably mental health challenges. The DASS-21 Assessment provides the link to respond to the DASS-21 Scales, while the DASS-21 scores gives the history of results of all student's DASS-21 Assessments. The DASS-21 scores should provide the trigger for immediate actions for a student whose situation might be critical. The other important components in the dashboard is notification. This can be seen in figure 3 .
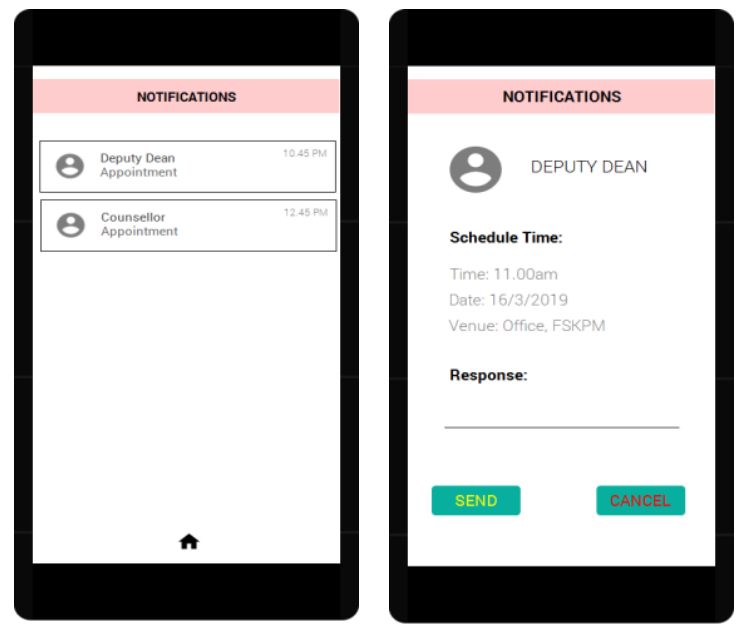

Fig. 4 Appointments \& notifications

\section{Modelling the Lecturer's View}

To start with, the most probable actors to notice a change in the attitude of a student are his or her lecturers. Hence, in this system, it was decided that lecturers should be assigned important responsibilities of paying keen attention to the participation of the students in their classes, specifically, they are required to observe students' attendance, grades, submission of assignments and their progress in class. If any anomaly behaviours below set thresholds are noticed with respect to any of the student's progresses, attendance or submission of assignments, the lecturers are obligated to refer the student (s) to the Deputy /Dean of Students' Affair or SWO via the Mental Health Monitoring System. Students whose anomaly behaviours are still within the set thresholds can be reminded by the lecturers themselves, to make necessary changes to improve in performance and overall class participation. The main features of the lecturer's dashboard are as seen in figure 5 .

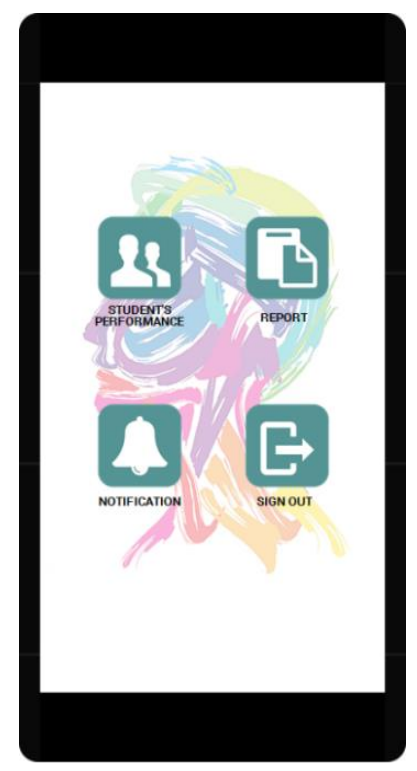

Fig. 5 Lecturer's Main Interface

D. Modelling the Deputy Dean or Dean of Student Affairs or Student Welfare Officer (SWO)'s View

The overall overseeing role in the mental health system rest on the SWO or the Admin-in-Charge person. Subsequently, schedule a meeting with the student. Based on the outcome of the meeting, it may be decided to refer the student for counselling or therapy or to schedule a session with a psychiatrist. The flow of Counsellor or 
Therapist/Psychiatrist is as seen in figure 8. Specifically, on receiving notification about a particular student, a thorough review of the student's reports such as recommendations and DASS-21 is expected to be carried out, which should guide as to whether to schedule a meeting with the student or not. If a meeting is conducted with the student, necessary freports and updates are provided for the Admin, or any other designated next of kin, as the case may warranty

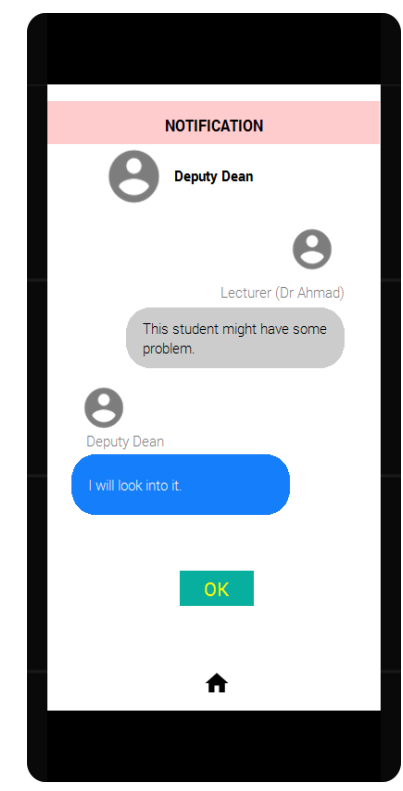

Fig. 6 Lecturer's Main Interface

The Counsellor or Therapist/Psychiatrist's tasks are to follow up on managing the students on reception of notifications from the SWO. They are required to schedule an appointment, diagnose the student's situation as well as provide appropriate interventions and treatments for the students. They are equally expected to update the SWO, for follow up and monitoring.

On receiving the notifications sent by lecturers, the SWO as seen in figure 6 , is expected to reach out to the student, extending invitation to the student to have a meeting. The purpose of this meeting is to clearly understand the situations or challenges that the student is having. Overall, the SWO continue to follow up and strive to resolve the student's problem. For students, whose conditions are severe, they are referred to the counsellors or therapists or psychiatrics for treatment. The flow of SWO's cause of actions is presented in figure 7 . This starts with reviewing notifications from the lecturers, followed by examining student's records and their respective DASS-21 scores. From this meeting, having examined the situations of the student properly, the student might be referred to counselling or therapist, while keeping track with the students' updates from counsellor or therapist to ensure positive progress and recovery. In a situation where the student does not respond to the SWO's invitation, then their next of kins, families or guardians are contacted to establish their situation.

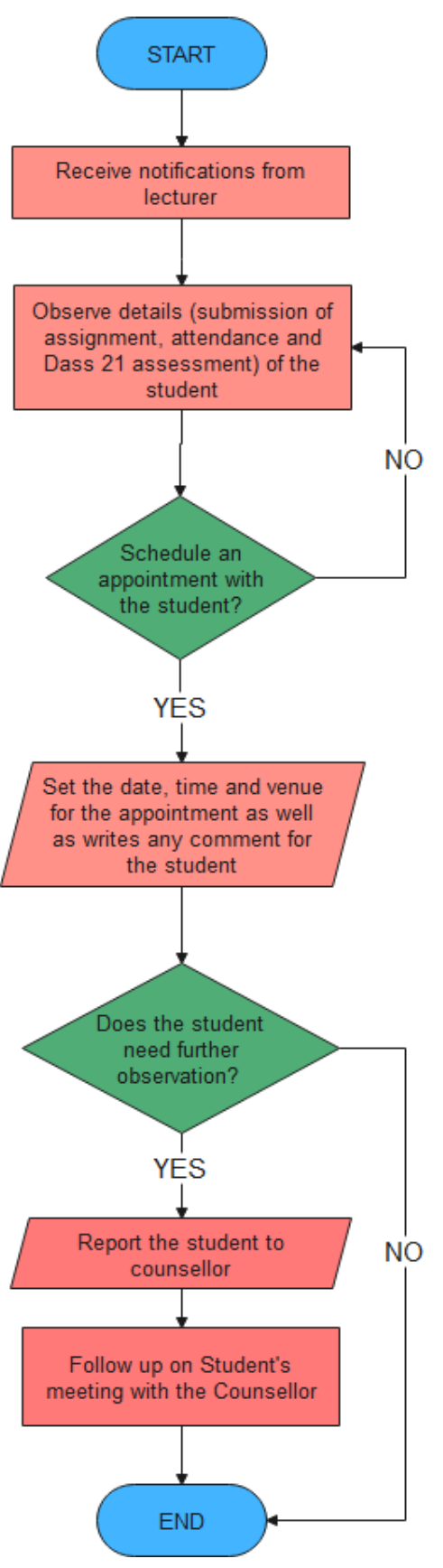

Fig. 7 University In-Charge Person or SWO's Flowchart 
E. Modelling the Counsellor or Therapist/Psychiatrist's View

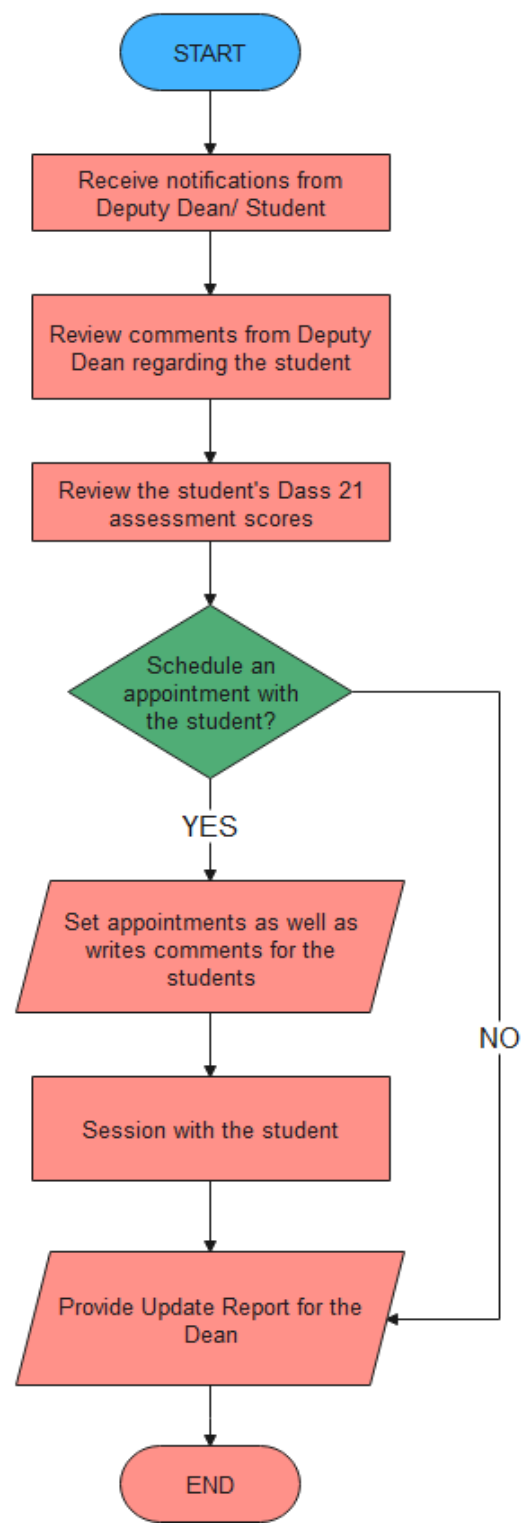

Fig. 8 Counsellor's Flowchart

\section{SYSTEM EVALUATION \& DISCUSSIONS}

Usability testing is used to assess the ease of use and the usability of a system. For this mental health monitoring system, several tasks required for each actor are broken down into manageable processes. Users are then given the tasks to test and explore the application. The usability testing processes are then observed by the developers or screen recorded for evaluation and system changes [40], [41]. This provides a means to check if the users are experiencing any difficulties while trying out the application with the given tasks. The time taken or duration for the users to complete each task is also recorded. From the recorded duration, the ease of use can be determined. Longer duration indicates that the users might be experiencing difficulties when utilizing the system. The next part of the usability testing is interview conducted with the users. This interview will give an opportunity for users to provide suggestions and feedbacks for improvement and appropriate modifications. Through this, the developers can also know the difficulties being faced by the users during the process of completing the tasks. After conducting the test and evaluation, feedback from the users can be utilised to better improve the remote mental health system's usability and functionality. The system can subsequently be published after the final improvements are made. Even after the system is published, further improvement can be improved by checking the users' feedback. Maintenance should be done frequently to satisfy the users' needs.

Most students do not have problem using the mental health monitoring system. They were able to use the system with ease and had good understanding of the overall system. However, there were some feedback from the students which were helpful to better improve this system for all the users. Most of them requested to have either side menus or bottom menus for easy access instead of going back to the homepage to access the menus in the dashboards. This is necessary to facilitate better navigation. Providing a menu for tracking or listing all the appointments set by the lecturers, SWO and counsellor/therapist is also one of the feedbacks given by the users. This will allow users to know and remind them of their appointments. Overall, the students think that this system will be helpful in assisting them in managing their mental health issues with the help or guidance from the SWO and counsellors.

Likewise, for lecturers, they were able to understand and use the system. However, they disagreed with the menu item name 'Report' which contains a list of student's reports of attendance, submission of assignments and DASS 21 assessment that have been sent to SWO. It is not a page to directly report a student to the SWO. The lecturers requested to change the name 'Report' to a different name that fits the function of the menu to avoid confusion when assessed. The counsellors encountered the same problem as the lecturers as well as SWO regarding the menu item name 'Report'.

The counsellors equally added that a consent letter should be provided for student when they are about to take the DASS-21 assessment because it is a very sensitive issue as well as a consent to allow lecturers, SWO and counsellors 
to view their results. If the students wish to continue in taking the DASS-21 assessment and agree to all the conditions, they will only then be allowed to do so. If they disagree, the students will not be allowed to answer the DASS-21 assessment. Meanwhile, counsellors emphasised that a through explanation should be displayed once they sign in into the system so that they can understand better how the system works.

Overall, majority of the users, namely, students, lecturers, SWO and counsellors supported the mental health monitoring system and agreed that it provides a better strategy to detect early and provide immediate care to the students.

\section{CONCLUSION}

Finally, this remote health monitoring system can be helpful to the students to manage their anxiety, stress and depression. Fast interventions can be given to the students by the university's in-charge persons and counsellors/therapists. Moreover, based on the usability testing and interviews conducted with the users, numerous feedbacks or recommendations were collected to improve the system. Based on the findings too, it is found that most of the students did not experience any major difficulty while testing out the system. Likewise, the SWO, lecturers and counsellors who were selected to test the system applauded it and hope that it would serve as a mean to improve mental health monitoring and caregiving among undergraduate students. Overall, most users suggested that this system might be very helpful to assist students in managing their mental states (stress, anxiety and depression) and provide opportunities for university administrations and caregivers to provide immediate help before the situation worsened.

A few challenges were envisaged based on human factors. Some students might answer the self-monitoring scales represented in the DASS 21 scales half-heartedly or ignore it altogether. They may take the self-monitoring scales as boring and thereby, full conscientious cooperation may not be guaranteed. The results might be inaccurate thus making the interventions from lecturers, universities' administration, or counsellors to be misleading. Maybe a guided response in the presence of the university administration such as the SWO may help.

Future work in this regard may include analysis of student's responses using text analysis as well as audio analysis to detect mental states. This may further provide better identification results to immediately identify those in need and respond accordingly.

\section{ACKNOWLEDGEMENTS}

The authors would like to acknowledge Universiti Malaysia Sarawak and the International Islamic University Malaysia for their supports for this research.

\section{REFERENCES}

[1] T. J. Legg, “Anxiety overview: Overview.," Healthline, 2016. .

[2] J. Brown, "Anxiety and Depression: Anxiety the most common mental health diagnosis in college students," BU Today, Oct-2016.

[3] R. Sani, "Coping with student mental health," New Straits Times, Apr2018.

[4] D. Rosenberg, " 1 in 5 college students have anxiety or depression. Here's why," The Conversation, 2018.

[5] C. N. Ka, C. Wang-Kin, and B. Y. F. Fong, "A Review of Academic Stress among Hong Kong Undergraduate Students," J. Mod. Educ. Rev., vol. 6, no. 8, pp. 531-540, , doi: 10.15341/jmer(2155-7993)/08.06.2016/003. 2016

[6] K. W. C. Lun et al., "Depression and anxiety among university students in Hong Kong," Hong Kong Med. J., vol. 24, no. 5, pp. 466472, doi: 10.12809/hkmj176915. 2018

[7] “Center for Collegiate Mental Health. (2016, January). 2015 Annual Report," 2016.

[8] Bernama, "Mental health of Malaysian students cause of worry: Minister health.," New Strait Times, Sep-2016.

[9] C. R. Doarn and R. C. Merrell, "Telemedicine, Telehealth, and the Public Health Good," Telemed. e-Health, vol. 25, no. 9, pp. 773-774, doi: 10.1089/tmj.2019.29028.crd. 2019

[10] J. Mathew, J. Lail, A. C. Chang, and J. L. Jefferies, Outpatient Monitoring and Self-Care. Elsevier Inc., 2018.

[11] A. M. Aldahmash, Z. Ahmed, F. R. Qadri, S. Thapa, and A. M Almuammar, "Implementing a connected health intervention for remote patient monitoring in Saudi Arabia and Pakistan: Explaining 'the what' and 'the how,"' Global. Health, vol. 15, no. 1, pp. 1-7, doi: 10.1186/s12992-019-0462-1. 2019

[12] G. Tse et al., "Telemonitoring and hemodynamic monitoring to reduce hospitalization rates in heart failure: A systematic review and meta-analysis of randomized controlled trials and real-world studies," J. Geriatr. Cardiol., vol. 15, no. 4, pp. 298-309, doi: 10.11909/j.issn.16715411.2018.04.008. 2018

[13] G. Aceto, V. Persico, and A. Pescapé, "The role of Information and Communication Technologies in healthcare: taxonomies, perspectives, and challenges," J. Netw. Comput. Appl., vol. 107, pp. 125-154, doi: 10.1016/j.jnca.2018.02.008. 2018

[14] "mHealth: New horizons for health through mobile technologies," World Health Organisation, 2018

[15] J. Fisher, D. T. Thach, and T. Tuan, "Validation of the depression anxiety stress scales (DASS21) as a screening instrument for depression and anxiety in a rural ... Validation of the depression anxiety stress scales (DASS) 21 as a screening instrument for depression and anxiety in a rur," BMC Psychiatry, vol. 13, no. 24, pp. 17, 2013

[16] D. L. Scher, "The Big Problem With Mobile Health Apps," Medscape, Mar-2015

[17] P. Chandrashekar, "Do mental health mobile apps work: Evidence and recommendations for designing high-efficacy mental health mobile apps," mHealth, vol. 4, no. 6, doi:10.21037/mhealth.2018.03.02. 
2018

[18] M. Fuller-Tyszkiewicz et al., “A mobile app-based intervention for depression: End-user and expert usability testing study," J. Med. Internet Res., vol. 20, no. 8, pp. 1-12, , doi: 10.2196/mental.9445. 2018

[19] M. T. Basco, R. E., \& Olea, "Correlation between anxiety level and academic performance of BS biology freshmen students," Int. J. Educ. Res. Technol., vol. 4, no. 1, pp. 97-103, 2013.

[20] R. Kaur, "Study of Stress among Adolescent Students in Relation to Working Status of their Mothers and family Environment," Educ. Quest- An Int. J. Educ. Appl. Soc. Sci., vol. 8, no. spl, p. 349, , doi: 10.5958/2230-7311.2017.00075.7. 2017

[21] J. M. G. Pariat, L., Rynjah, A., \& Kharjana, "Stress levels of college students: Interrelationship between stressors and coping strategies.," J. Humanit. Soc. Sci., vol. 19, no. 8, pp. 40-46, 2014.

[22] L. M. Thawabieh, A. M., \& Qaisy, "Assessing Stress among University Students," Am. Int. J. Contemp. Res., vol. 2, no. 2, pp. 110-116, 2012

[23] R. I. El-Ezaby, H. H., Elalem, O. M., \& El-Mowafy, "Factors affecting depression among teenagers in Port Said City," J. Nurs. Heal. Sci., vol. 4, no. 1, pp. 12-18, doi: 10.9790/1959-04131218. 2015

[24] N. K. Bhasin, S. K., Sharma, R., \& Saini, “Depression, anxiety and stress among adolescent students belonging to affluent families: $A$ School-based study," Indian J. Pedatrics, vol. 77, doi: 10.1007/s12098009-0260-5. 2010

[25] P. R. Albert, "Why is depression more prevalent in women?," Jorunal of Psychiatry Neurosci, vol. 40, no. 4, pp. 219-221, , doi: 10.1503/jpn.150205. 2015

[26] C. Hammen, "Stress and Depression," Annu. Rev. Clin. Psychol., vol. 1, pp. 293-319, 2005.

[27] M. T. Sarokhani, D., Delpisheh, A., Veisani, Y., \& Sarokhani, "Prevalence of Depression among University Students: A Systematic Review and Meta-Analysis Study," Depress. Res. Treat., doi: $10.1155 / 2013 / 373857.2013$

[28] A. Goff, "Stressors, academics performance, and learned resourcefulness in baccalaureate nursing students.," Int. J. Nurs. Educ. Scholarsh., vol. 8, no. 1, pp. 1-20, 2011.

[29] A. A. Turner DP, Thompson ME, Huber LR, "Depressive symptoms and academic performance of North Carolina college students," N. C. Med. J., vol. 7, no. 3, pp. 169-175, 2012.
[30] A. Islam, M. A., Low, W.Y., Tong, W. T., Yuen, C. C. C., \& Abdullah, "Factors Associated with Depression among University Students in Malaysia: A Cross-sectional Study," in The 2nd International Meeting of Public Health 2016, 2018.

[31] J. Moran, J., Kelly, G., Haberlin, C., Mockler, D., \& Broderick, “The use of eHealth to promote physical activity in patients with mental health conditions: a systematic review," HRB Open Res., , doi: 10.12688/hrbopenres.12796.1. 2018

[32] F. Gravenhorst et al., "Mobile phones as medical devices in mental disorder treatment: an overview," Pers. Ubiquitous Comput., vol. 19, no. 2, pp. 335-353, , doi: 10.1007/s00779-014-0829-5. 2015

[33] S. J. Naslund, J. A., Marsch, L. A., Mchugo, G. J., \& Bartels, “Emerging mHealth and eHealth interventions for serious mental illness: a review of the literature," J. Ment. Heal., vol. 24, no. 5, , doi: 10.3109/09638237.2015.1019054. 2015

[34] G. Luxton, D. D., Bush, N., \& Reger, "mHealth for mental health: Integrating smartphone technology in behavioral healthcare," Prof. Psychol. Res. Pract., vol. 42, no. 6, pp. 505-512, , doi: 10.1037/a0024485. 2011

[35] G. Labrique, A., Vasudevan, L., Kochi, E., Fabricant, R., \& Mehl, "mHealth innovations as health system strengthening tools: 12 common applications and a visual framework," Glob. Heal. Pract. J., vol. 1, no. 2, 2013

[36] J. Kuhn, E., Greene, C., Hoffman, J., Nguyen, T., Wald, L., Schmidt, J., Ramsey, K. M., \& Ruzek, "Preliminary Evaluation of PTSD Coach, a Smartphone App for Post-Traumatic Stress Symptoms," Mil. Med., vol. 179, no. 1, pp. 12-18, , doi: 10.7205/MILMED-D-13-00271. 2014

[37] S. Ehrenreich, B., Righter, B., Rocke, D, A., Dixon, L., \& Himelhoch, "Are mobile phones and handheld computers being used to enhance delivery of psychiatric treatment?: a systematic review," J Nerv Ment Dis, vol. 199, no. 11, pp. 886-891, 2011.

[38] V. Harrison, V., Proudfoot, J., Wee, p. P., Parker, G., Pavlovic, D. H., \& Manicavasagar, "Mobile mental health: Review of the emerging field and proof of concept study," J. Ment. Heal., vol. 20, no. 6, pp. 509524, 2011, doi: 10.3109/09638237..608746. 2011

[39] K. Malasinghe, L., Ramzan, N., \& Dahal, "Remote patient monitoring: a comprehensive study," J. Ambient Intell. Humaniz. Comput., 2017.

[40] Euphemia Wong, "Schneiderman's Eight Golden Rules Will Help You Design Better Interfaces.," The Interaction Design Foundation, 2018

[41] "How to conduct a cognitive walkthrough," Interaction Design Foundation, 2018 